\title{
Enhancement of Medical Image Fusion Using Image Processing
}

Dr. U. Palani

\author{
Professor
}

Department of ECE

IFET College of Engineering

Villupuram, India

palani_uin@yahoo.com

\section{Mrs. D. Vasanthi}

Associate Professor

Department of ECE

IFET College of Engineering

Villupuram, India

dvasanthime@gmail.com

\author{
Ms. S. Rabiya Begam \\ UG Student \\ Department of ECE \\ IFET College of Engineering \\ Villupuram, India \\ fazwinfadheela2000@gmail.com
}

\begin{abstract}
The assortment of all indispensable data from at least two pictures and the principle incorporation is partitioned into less picture pixels as a rule into an individual one to build the norm of the picture and diminish the iterative so as to improve all the fundamental uses of clinical picture which is utilized to investigate all the clinical issues. This cycle is called as Clinical picture mixture. The parceling by watershed calculation for these sub-pictures are standard to get the areas of individual level, these districts are utilized to manage the combination cycle. To keep up the basic enumerations, the watershed calculation is accustomed to achieving the picture division.
\end{abstract}

Keywords - assortment, watershed, enumerations.

\section{INTRODUCTION}

These days, communicated software engineers center around advanced information and screening methods are intended for recognizing early side effects of disease and change for treatment. In clinical explain, there are different cycle with different capacities to get profitable information on human life systems. Despite the fact that, there might be parcel of sources, a solitary information can't give more significant data in breaking down ailments. The issue with single source is expensive, fastidious, causing mistake, tedious and it requires more understanding. These components of single picture roused the scientists to get fitting data. Besides, the most recent picture modalities are all the more exorbitant, which are the extra weight for the people. Along these lines, it is imperative to amalgamate an every single pictures into an individual picture, which is more appropriate for useful symptomatic assessment. Among a few serious ailments malignant growth and cerebrum tumor are the most compromising infection to the humankind. It is exceptionally simple to distinguish and fix the malignancy and cerebrum tumor cell in beginning stage, however in the event that they gets progressed, at that point it will be hard to treat. PET (positron emanation tomography) is utilized to see the metabolic conditions in the various pieces of the body. X-ray (extent reverberation picture) is utilized to recognize the delicate bone tissues over the body and 
Journal of Innovative Image Processing (JIIP) 2020)

Vol.02/ No. 04

Pages: $165-174$

https://www.irojournals.com/iroiip/

DOI: https://doi.org/10.36548/jiip.2020.4.001

comparatively CT (figured tomography) distinguishes the hard tissues over the body. We will takes these two pictures (PET and X-ray), which go through combination cycle, and gives more data for the conclusion. A tumor is characterized as the new development of uncommon tissue that is regularly surrendered and open minded. The tumor cells do develop, regardless of whether the body doesn't need them and not at all like old cells, these cells don't pass on effectively causing disease or tumor. As the cycle goes (i.e., as the tumor cells develop on) the tumor keeps on developing and an ever increasing number of cells are added to mass. There are two sorts of tumors in particular, malignant tumors and non-harmful tumors.

Clinical figure blend encompasses a wide scope of techniques from picture combination and general information to address clinical issues reflected through pictures of human organs, life systems, and cells. There is a developing interest and utilization of the imaging innovations in the regions of clinical perceiving, investigation and chronicled confirmation. Since PC supported imaging strategies empower a distinguishable assessment of the likeness under assessment, it assists with improving the reasonableness of the clinical professionals in showing up at an unbiased and target choice in a limited capacity to focus time. This regularly prompts powerful data handling that can examine the data that is generally undetectable to natural eyes. The additional data gained from the amalgamated pictures can be all around used for more exact pinpoint of diaphragm.

\section{RELATED WORK}

A basic information gave by the clinical picture to dissect the ailment can be improved through picture combination utilizing different modalities. This paper presents the combination cycle which permits the appropriation highlights of various methodology pictures. It additionally comprises of Head Part Analysis(PCA) which is utilized to decide the metabolic exercises and this prompts recognize the tumor discovery through picture fusion. The framework characterized underneath covers the PCA and DCT based picture combination standard examination for dissecting of malignant growth tumor and the exploratory outcomes gives the viability of combination rule dependent on head segment investigation and discrete cosine change which identifies the first basic information from source picture and furthermore improves the pertinent data from the equivalent [1]. The triumphant conclusion of a sickness relies upon the norm of the picture got from clinical imaging procedure. The objective of clinical imaging is to procure a high goal picture with more data for analytic purposes. This paper proposes a half and half combination rule for multimodality clinical pictures. There are two kind of systems one is 'Life systems', which gives basic subtleties of body parts, for example, X-beam, PET, X-ray, and other 'Physiology and Digestion', it gives the information about useful subtleties of life structures movement in the body parts, for example, SPECT, PET. Subsequently, both of the Life structures, Physiology and Digestion pictures are inspected [2]. Specialists utilize clinical pictures to discover irregularities in human life structures and to find the interruptions. In transmission strategy in some cases clinical pictures are defiled because of ecological clamor. Along these lines need to improve the picture standard, put down the computational intricacy and sign to clamor proportion (SNR). There is need of division to expand the presentation examination and picture standard. Division strategy is utilized to distinguish significant locales in clinical pictures, it is an exceptional method for apportioning picture into important sub districts or item with same trait. Proposed technique state Guideline part examination and k-implies grouping is a cycle of quantization, initially from preparing of sign which is well known for mining .Including Rule Segment Investigation for highlight extraction and development of exact number of bunch to build the precision [3]. In Existing framework, the mix of DWT and Head part examination (PCA) picture combination of mind tumor identifying tissues. The X-ray and PET pictures are dealt with into discrete wavelet change. Each information after the cycle of division now every picture has an each element advancement which is utilized to investigation and acknowledgment of the picture. The discrete wavelet change is a sort of change whatever wavelets are examined as discrete. Primary preferred position is that it performs Fourier change and express the recurrence and area in time. Here can't straightforwardly apply discrete wavelet change thus, sending into red, green, and blue of the info picture individually. For lower coefficients follows the low pass channel, lower higher coefficients follow the vertical astute limit line division, higher lower coefficients follows the even savvy division, higher coefficients follow the inclining insightful division. From the outset phase of dwt is to isolate the recurrence sub groups from pictures to perform tasks like multi scale and multi-goal strategies that to meet its scale amount. Combination strategy for two information picture is finished by twodimensional dwt. It have the multi scale goal of inaccessible divisions of a picture with different frequencies. 
Journal of Innovative Image Processing (JIIP) 2020)

Vol.02/ No. 04

Pages: $165-174$

https://www.irojournals.com/iroiip/

DOI: https://doi.org/10.36548/jiip.2020.4.001

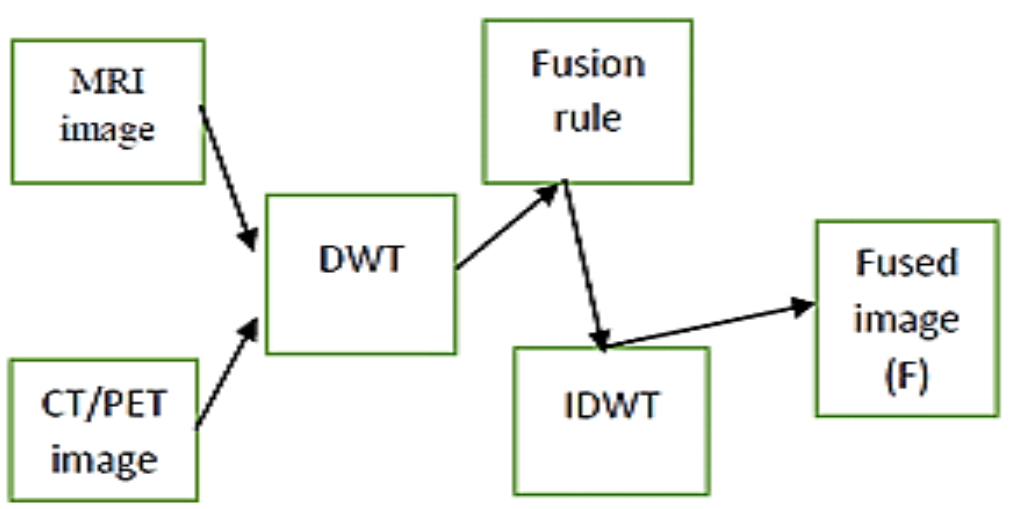

FIG.1 DWT

Stage 1:- The info multi scale pictures, whichever goes through combination measure, must be selected for the comparing pixels alteration.

Stage 2:- The X-ray and PET pictures are dealt with into discrete wavelet change. Here these pictures might be high sub groups or low sub groups as clarify in overhead discrete wavelet change.

Stage 3:- One of the combination rule is applied and subsequently coefficients will get of different pieces of an information pictures.

Stage 4:- Subsequent to acquiring the coefficients in above advance utilizing those which can apply the IDWT to get the last combined picture. The perfection and sharpness qualities of the picture consistently in lower band of picture, lower consistently conveys the significant data with them.

\section{A. PRINCIPAL COMPONENT ANALYSIS (PCA)}

The another name for the PCA is Hoteling change or Karhunen-Loeve change. It gives the upgraded information from the underlying pictures. PCA is a logical arrangement which changes over the corresponded into the nonrelated factors. In the PCA, the underlying segment is constantly planned along towards high difference.

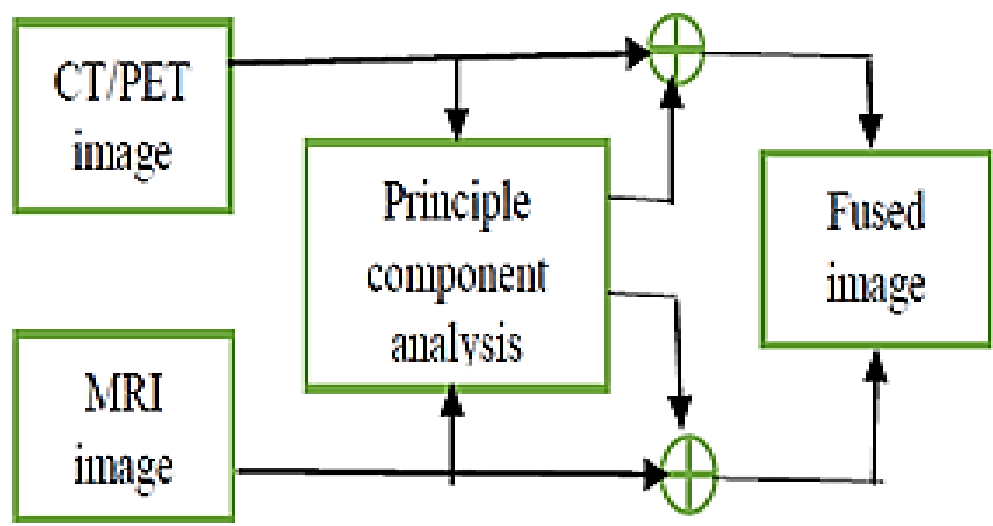

FIG.2 PCA 
Journal of Innovative Image Processing (JIIP) 2020)

Vol.02/ No. 04

Pages: $165-174$

https://www.irojournals.com/iroiip/

DOI: https://doi.org/10.36548/jiip.2020.4.001

The resulting segment is ordinary to the essential. The accompanying advances is followed for PCA

Step1:- The two info pictures CT and X-ray are organized segment vectors.

Step2:- Ascertain size of the info date and locate the mean and deduct the from input information.

Step3:- Subsequent to deducting, the mean introductory information at that point legitimately discover Eigen esteems from it.

Step4:- This end vector will assesses the liking and varieties. This Eigen vectors regarding the more Eigen esteem is received.

\section{PROPOSED SYSTEM}

The info picture 1 and information picture 2 are melded by division utilizing watershed calculation. Middle channel is utilized to diminish the natural commotion in a picture. The watershed calculation is a device morphological based for picture partioning. Division calculation assumes a significant job in locale based picture blend technique. Highlights ought to be sectioned as on its own locales. The rule point of the picture upgrade is to deal with a picture for a particular assignment so the handled picture is preferable seen over the past picture. Preprocessing is measure which causes us to separate the foundation picture from the forefront picture which is liberated from commotion. Power of the picture ought to be standardized. By assessing an information picture or picture caught by advanced camera, is to evacuate the foundation interruptions, picture can get improved visual appearance of information pictures.

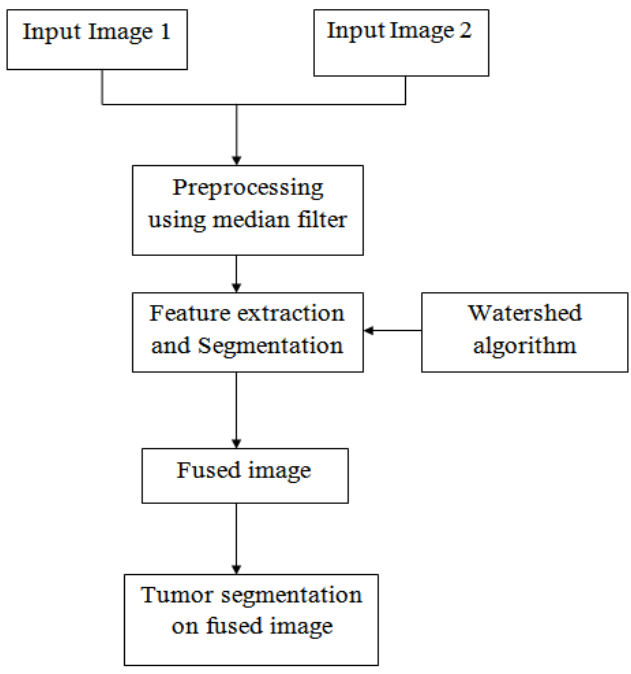

FIG.3 FLOWCHART

\section{A. MEDIAN FILTER}

The Middle channel is a non-risk arithmetical sifting method, regularly used to expel outer aggravations from a picture or sign. Such clamor decrease is a common pre-treatment venture to improve the aftereffects of later handling. The middle channel endeavors by traveling through the picture pixel by pixel, supplanting each an incentive with the middle estimation of an adjoining pixels. The middle is controlled by first organizing all the pixel evaluations from the window into irregular request, and afterward moving the pixel being considered with the inside (middle) estimation of a picture pixel.

\section{B. SEGMENTATION}

Division is the way toward parting an advanced picture into different fragment esteems. The consequence of picture parting is a lot of fragments that altogether spread the entire picture, or a lot of shapes acquired from a 
Journal of Innovative Image Processing (JIIP) 2020)

Vol.02/ No. 04

Pages: $165-174$

https://www.irojournals.com/iroiip/

DOI: https://doi.org/10.36548/jiip.2020.4.001

picture pixels. Every one of the pixels in an area is comparative as for some trademark or diagnosing property, for example, shading, force, or surface. The division step was utilized to isolate the picture into various locales dependent on comparative sanctioning in the picture. These locales show particular and exceptional attributes from each other and ought not converge each other. Every one of the districts ought to depict some degree of consistency in the area. In picture preparing, division falls into the class of getting different picture properties of a unique picture.

\section{FEATURE EXTRACTION}

Highlight extraction is the most productive piece of this paper. The properties standard deviation, entropy, differentiate and so on are extricated from the picture and are utilized to prepare the dataset for the grouping. Picture change is the examination and utilization of graphical pictures from sources, for example, pictures and recordings. There are three vital strides in picture upgrade; first, is the transformation of caught pictures into computerized esteems that a PC can measure; second, is the picture extraction and information pressure, and the third is the last advance that comprises of the presentation by the handled picture. The most essential area of this division technique is expansion of highlight locale. Be that as it may, in genuine pictures, commotion is harming the picture worth or picture ordinarily comprises of finished portions. This outcome is the upgrade of the distinguished bug bother from the picture pixel. The last picture which was gotten toward the finish of the past stage was utilized in this stage. The picture pixel estimations of the yield picture will be checked both vertically and evenly to discover the directions in the picture. The broadness and tallness of the removed picture was dictated by utilizing its beginning and consummation arrange values.

\section{WATERSHED ALGORITHM}

Division calculation assumes a significant job in locale based picture combination measure. Highlights ought to be fragmented as single one of a kind districts. Highlight might be partitioned into more than one area and individual district must be dealt with independently. On the off chance that conceivable, less number of areas ought to be created to diminish the computational weight. Picture combination is an element level picture combination in which picture is divided to districts and the comparing areas are amalgamated. Highlight is fundamental than the single picture pixel. Subsequently it is smarter to fuse the component data in the combination cycle. Division calculation plays a significant activity in locale based picture blend measure. Highlights ought to be fragmented as novel locale. Highlight might be partitioned into more than one district and individual locale must be dealt with independently. On the off chance that conceivable, low level districts ought to be created to diminish the computational weight.

Watershed Calculation has the accompanying advances :

1. Computing a division work. This is the pixel esteem whose dim areas are the items to be portioned.

2. Compute closer view markers. These are joined masses of pixels inside every one of the items.

3. Ascertain foundation markers. These are pixels that are not part of any unique picture.

4. Adjust the division work with the goal that it has diminished at the frontal area and foundation marker territories.

5. Ascertain the watershed change of the adjusted portioned esteem. The watershed calculation straightforwardly acquire the outcomes in over division measure. This over division can be disregarded utilizing marker controlled watershed calculation. In district based picture combination technique, the pictures to be melded ought to be divided. There ought to be a solitary division map for both the pictures. It could be conceivable by consolidating the each divided picture esteems.

\section{IMPLEMENTATION}

In the desk work, the analyses are conveyed utilizing Mat lab programming. We have arranged an execution with expanding the estimation of entropy in an information pictures which gives precise outcomes in the intertwined picture. Choosing on every menu will play out a different rating picture pixels. Computerized picture preparing, as an apparatus to dissect the shading and concentrate the essential qualities of a picture. The model innovation needs more precise outcomes for that we recognize the mind tumor by expanding the entropy

ISSN: 2582-4252 (online)

Submitted: 20.09 .2020

Accepted: 15.10.2020

Published: 21.10 .2020

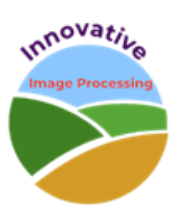


Journal of Innovative Image Processing (JIIP) 2020)

Vol.02/ No. 04

Pages: $165-174$

https://www.irojournals.com/iroiip/

DOI: https://doi.org/10.36548/jiip.2020.4.001

and complexity esteems for PET pictures by(0.1). It is an improvement of authentic data for human understanding and handling of picture information for capacity, transmission, and order for self-governing machine perspective.

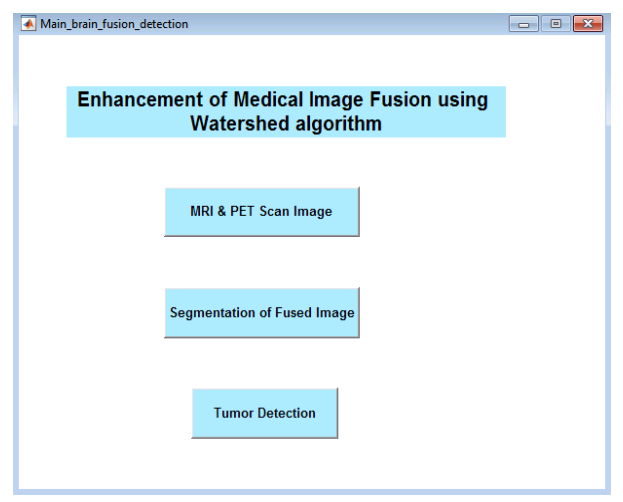

FIG.4 OUTPUT GUI WINDOW

This is the output execution window after running the program which contains the overall process of the brain tumor detection. The GUI window is used to distinguish graphical interfaces from text based ones, such as command line interfaces.

MRI is taken as the first input image which uses a large magnet and radio waves to look at organs and structures inside our body.

PET is taken as the second input image which uses the radioactive substances to visualize and measure metabolic processes in the body. PET is mainly used in the area of medical imaging for detecting or measuring changes in physiological activities like metabolism, blood flow, absorption and therefore called as a functional imaging technique.

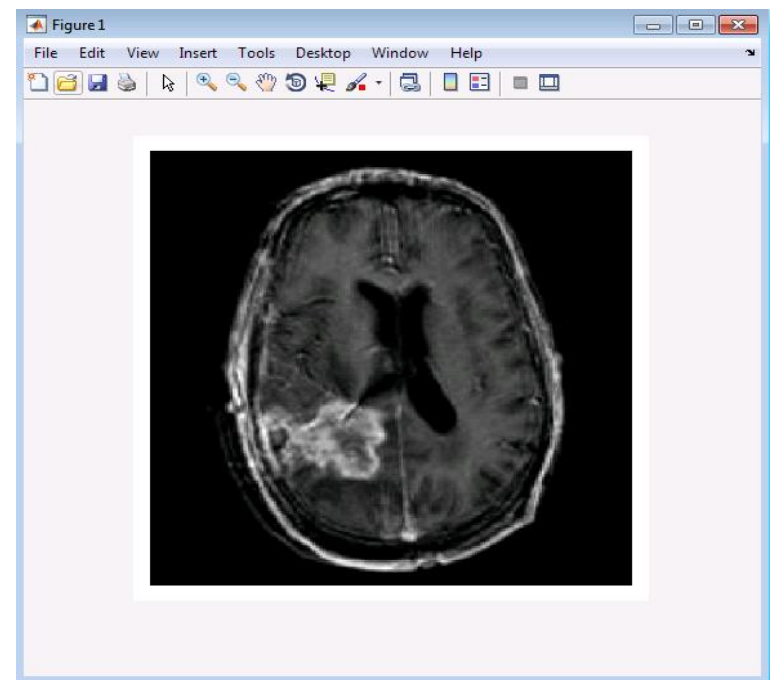

FIG.5 MRI IMAGE(input 1) 
Journal of Innovative Image Processing (JIIP) 2020)

Vol.02/ No. 04

Pages: $165-174$

https://www.irojournals.com/iroiip/

DOI: https://doi.org/10.36548/jiip.2020.4.001

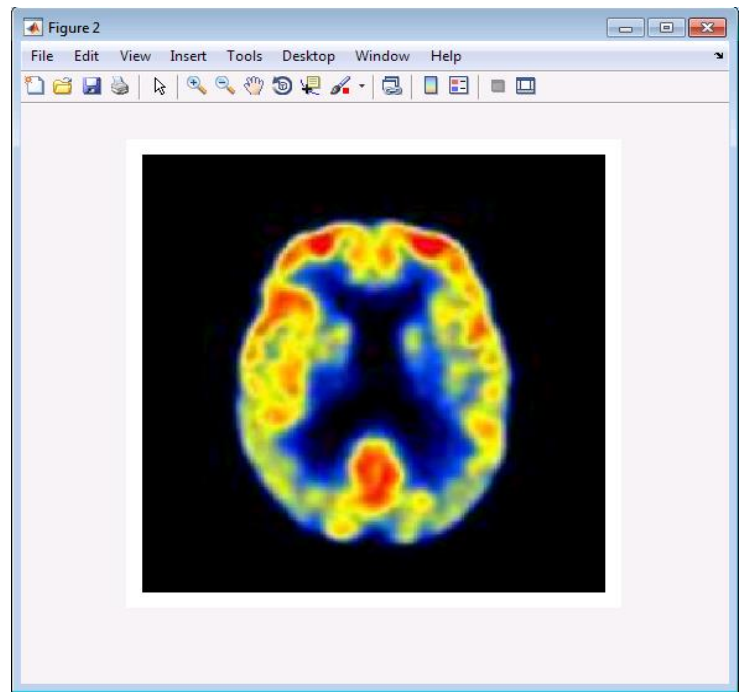

FIG.6 PET IMAGE (input 2)

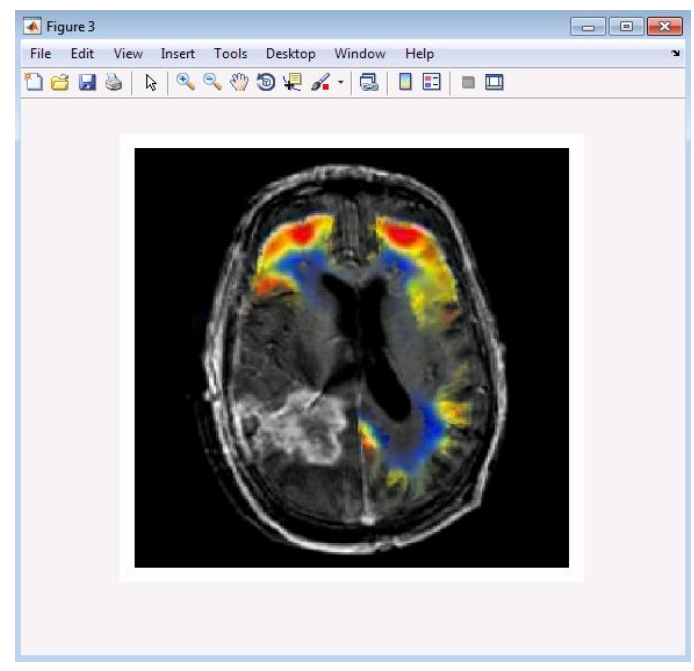

FIG.7 FUSED IMAGE

The combination of both Black and white (MRI) and colored image (PET) gives the fused image which gathers all the important information from multiple images, and their inclusion into fewer images, usually a single one. This single image is more informative and accurate than any single source image, and it consists of all the necessary information. It is more appropriate and understandable for the human and machine perception. 
Journal of Innovative Image Processing (JIIP) 2020)

Vol.02/ No. 04

Pages: $165-174$

https://www.irojournals.com/iroiip/

DOI: https://doi.org/10.36548/jiip.2020.4.001

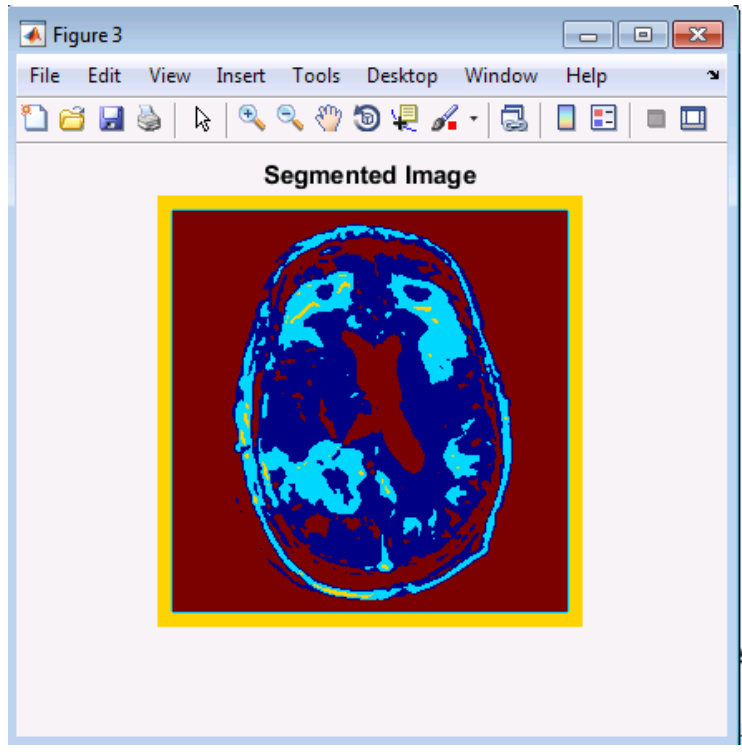

FIG.8 SEGMENTED IMAGE

Image segmentation is the process of partitioning a digital image into multiple segments (sets of pixels).The goal of segmentation is to simplify and/or change the representation of an image into something that is more meaningful and easier to analyze. It is typically used to locate objects and boundaries in an images and also used to assign a label to every pixel in an image.

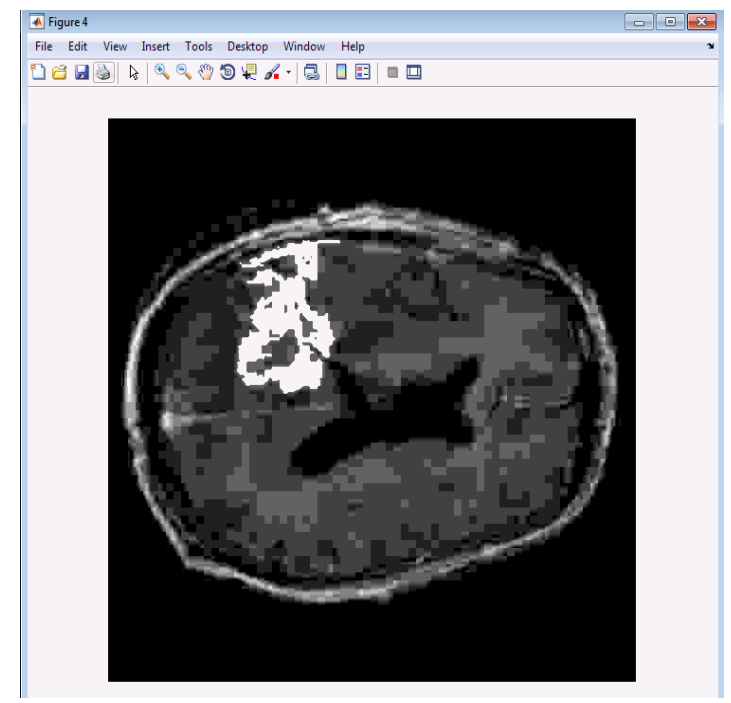

FIG.9 TUMOR REGION

The segmented image which does not contains any noise removed by median filter. Such noise reduction in a typical preprocessing step to improve the results of later processing. Median filtering is very widely used in digital image processing because, under certain conditions, it preserves edges while removing noise, also having applications in signal processing. This is the final output image after considering the two input images. The noise can be reduced by median filter after that segmentation, the partitioning of an image can be detected by the Watershed algorithm. Finally the tumor will be detected accurately by the doctors and it will be helps to rectify the problems of the affected person (brain tumor) as earlier as possible. 
Journal of Innovative Image Processing (JIIP) 2020)

Vol.02/ No. 04

Pages: $165-174$

https://www.irojournals.com/iroiip/

DOI: https://doi.org/10.36548/jiip.2020.4.001

Formula used:

Mean:- ${ }^{-}=\sum x N$

Entropy: $-\sum P L-1 L=0$ i $\log 2 \mathrm{Pi}$

Standard deviation:- $1 N-1 \sum(x N-1 i=0 \mathrm{i}-\mu) 2$

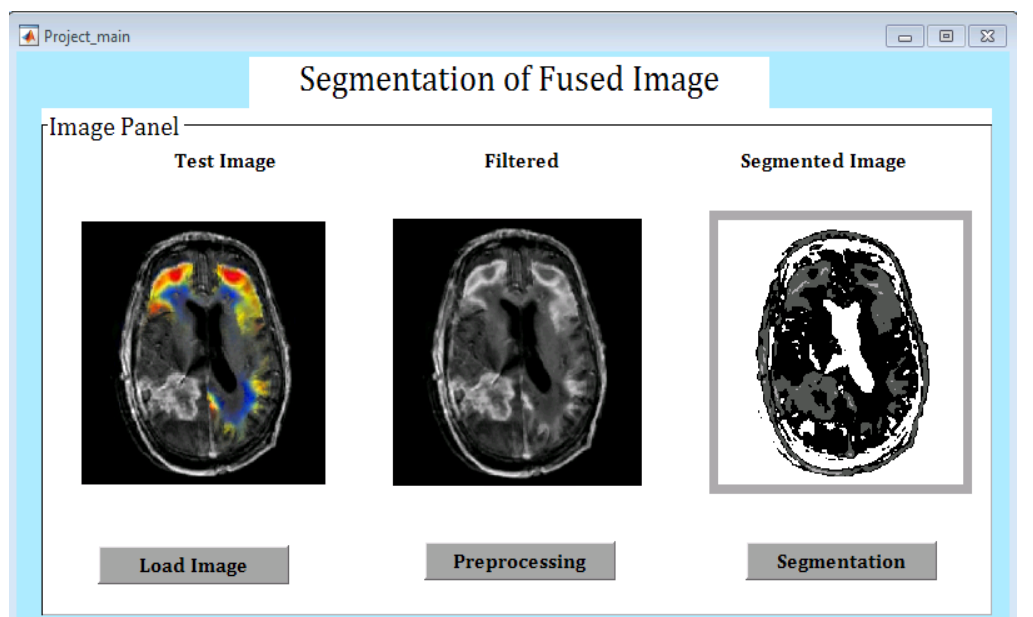

PARAMETER EVALUATION:

\begin{tabular}{|c|c|c|c|}
\hline & $\begin{array}{c}\text { MRI } \\
\text { IMAGE }\end{array}$ & $\begin{array}{c}\text { PET } \\
\text { IMAGE }\end{array}$ & $\begin{array}{c}\text { FUSED } \\
\text { IMAGE }\end{array}$ \\
\hline MEAN & 0.1654 & 0.2235 & 0.2998 \\
\hline ENTROPY & 6.4892 & 6.6489 & 7.8653 \\
\hline SD & 0.1786 & 0.2869 & 0.2876 \\
\hline
\end{tabular}

\section{CONCLUSION}

The picture combination of X-ray and PET pictures is acted in MATLAB GUI. From the reproduction result, it is dubious that combined picture is having the norm of both the source input esteems. The combined picture having an away from of the tumor is taken as the information picture for apportioning utilizing watershed technique and tumor cells are divided and separated from foundation without losing of any info esteems. The fundamental point of the task is to get more data on combination utilizing two modalities of pictures and the irregularity in the source picture (i.e.) tumor is portioned from the intertwined picture which encourages the specialist to dissect the tumor as prior as could reasonably be expected, outline the anatomical and physiological contrasts starting with one dataset then onto the next and evade superfluous medical procedures.

\section{FUTURE WORK}

Future work additionally prompts mirror the combination idea in different applications in different fields. To support specialists and doctors in different applications incorporate estimating separation between bone district and unusual area, to gauge tumor thickness and size from yield picture. Combination rule can be extended to different information source pictures like PET/X-ray combination and thought with typical picture from PET/CT scanner where the enormous measure of cost has been diminished. To actualize the combination cycle for ongoing applications which obtained from the different symptomatic investigation from the medical clinic. 
Journal of Innovative Image Processing (JIIP) 2020)

Vol.02/ No. 04

Pages: $165-174$

https://www.irojournals.com/iroiip/

DOI: https://doi.org/10.36548/jiip.2020.4.001

\section{REFERENCE}

[1] Rohit Kempanna Atyali, and Shivchandra R Khot, "An Enhancement In Detection Of Brain Cancer Through Image Fusion,” 2016 IEEE International Conference on Advances in Electronics, Communication and Computer Technology (ICAECCT),pp.438- 442.

[2] Guruprasad S, M Z Kurian and H N Suma "Fusion of CT and PET medical images using hybrid algorithm DWT-DCTPCA" 978-1-4673-8611-1/15 2015 IEEE.

[3] Samet Aymaz, Cemal Kose, "Multi focus image fusion using stationary wavelet transform(SWT) with principle component analysis(PCA)", 2017, pp $1176-1180$.

[4] Sindhuja madanala and K Jhansi Rani, "PCA-DWT based medical image fusion using non sub sampled counterlet transform", international conference on signal processing, communication, power and embeded system (SCOPES) - 2016.

[5] Arpita Das and Mahua Bhattacharya " Effective Image Fusion Method to Study Alzheimer's Disease using MR, PET Images" 2015 IEEE International Conference on Bioinformatics and Biomedicine (BIBM),pp-16031607.

[6] V.Gomathy and U. Snekhalatha "Automated segmentation using PCA and area estimation of thyroid gland using ultrasound Images" IEEE Sponsored 2nd International Conference on Innovations in Information Embedded and Communication Systems ICllECS'15.

[7] Dr. Hema Menon P. and Narayanankutty, K. Ab, "MRI/CT image fusion using Gabor texture features", in Advances in Intelligent Systems and Computing, 2016, vol. 530, pp. 47-60. 